\title{
Attitude Control System Design of UAV
}

\section{Guo $\mathrm{Li}^{1, \mathrm{a}}$, Xiaoliang Lv ${ }^{2, \mathrm{~b}}$, Yongqing Zeng ${ }^{3, \mathrm{c}}$}

\author{
${ }^{1}$ Automation School, University of Science and Technology Beijing, Beijing, 100083, China \\ ${ }^{2}$ Automation School, University of Science and Technology Beijing, Beijing, 100083, China \\ ${ }^{3}$ Automation School, University of Science and Technology Beijing, Beijing, 100083, China \\ aemail: guo6393@sina.com, bemail: Iv_maxwell@sina.com, ${ }^{\text {cemail: } 1454848644 @ q q . c o m}$
}

Keywords: Nonlinear; ANNs; Predict Model; Attitude Control; Simulation.

\begin{abstract}
Based on the characteristics of the attitude control system, this paper use the neural network predictive model controller which can identify accurately attitude system and obtain real-time control sequence applied to the controlled object through the model prediction, feedback correction and rolling optimization each link to complete motion attitude control of unmanned aerial vehicle. This control system has good control effect, meanwhile, the fast, accuracy and anti-disturbance capability tracking a given process can be effectively improved.
\end{abstract}

\section{Introduction}

There is a very significant piece of legislation that attitude control is extremely important for performance of the unmanned aerial vehicle (UAV) flight system. However, Movement of UAV attitude in the actual process contains a lot of uncertain factors. To get a higher control precision, we only use more advanced control theory. In this paper, first of all, posture model was established through simplifying scientifically. Secondly, combining with the expected control goal and the actual conditions, we adopt the prediction algorithm based on BP neural network in the design and expect to achieve better control effect. Then in order to better show the advanced design performance of the controller, we will compare and analyze with the classic PID control performance. Finally, the system performances of robust and adaptability were studied and analyzed.

\section{System Model}

In order to study conveniently, when establish UVA's model [1], we have the following two assumptions: aerial robot is rigid and quality is constant; ignore the ground curvature and the influence of the gravitational acceleration in model.

$$
\begin{aligned}
& \dot{P}=V \\
& m \dot{V}=C_{e}^{b} * f b+\left[\begin{array}{lll}
0 & 0 & m g
\end{array}\right] T \\
& \dot{R}=H \omega b \\
& \text { Inside, } H=\left[\begin{array}{ccc}
1 & S \varphi T \theta & C \varphi T \theta \\
0 & C \varphi & -S \varphi \\
0 & S \varphi / C \theta & C \varphi / C \theta
\end{array}\right], \text { So attitude angle motion equation is following: } \\
& \left\{\begin{array}{l}
\frac{d \theta}{d t}=q \cos \varphi-r \sin \varphi \\
\frac{d \varphi}{d t}=p+\tan \theta(q \sin \theta+r \cos \varphi) \\
\frac{d \psi}{d t}=(q \sin \varphi+r \cos \varphi) / \cos \theta
\end{array}\right.
\end{aligned}
$$


Attitude kinetic equation by the moment of momentum theorem is:

$J \dot{\omega} b+\omega b \times J \omega b=t b$.

Inside, inertia matrix is: $J=\left[\begin{array}{ccc}I x x & 0 & -I y y \\ 0 & I y y & 0 \\ -I x x & 0 & I z z\end{array}\right]$.

Instead of blade element theory, we use straight the way of ideal moment research to simplify the system model, ignore coupling effects between main rotor and tail rotor. Theory suggests that this kind of approximation is still meaningful within a certain range in study [2]. By the motion equation Eq.4, Eq.5, selected $\left[\begin{array}{llllll}w_{x} & w_{y} & w_{z} & \phi & \theta & \psi\end{array}\right]^{T}$ as state variables, the initial state is $\mathrm{R}=[0 ; 0 ; 0]$, the state space expression can be obtained.

$\left\{\begin{array}{l}\dot{x}=A x+B u \\ y=C x+D u\end{array}\right.$

Inside, $A=\left[\begin{array}{cccccc}0 & \frac{J_{Z}-J_{S}}{J_{1}} & \frac{J_{Z}-J_{S}}{J_{1}} & 0 & 0 & 0 \\ \frac{J_{S}-J_{1}}{J_{Z}} & 0 & \frac{J_{S}-J_{1}}{J_{Z}} & 0 & 0 & 0 \\ \frac{J_{1}-J_{Z}}{J_{S}} & \frac{J_{1}-J_{Z}}{J_{S}} & 0 & 0 & 0 & 0 \\ 1 & 0 & 0 & 0 & 1 & 0 \\ 0 & 1 & 0 & -1 & 0 & 0 \\ 0 & 0 & 1 & 1 & 0 & 0\end{array}\right], B=\left[\begin{array}{cccccc}\frac{1}{J_{1}} & \frac{1}{J_{2}} & \frac{1}{J_{3}} & 0 & 0 & 0\end{array}\right]$

$C=\left[\begin{array}{llllll}0 & 0 & 0 & 1 & 0 & 0 \\ 0 & 0 & 0 & 0 & 1 & 0 \\ 0 & 0 & 0 & 0 & 0 & 1\end{array}\right], \quad D=0$.

\section{Controller Design}

\subsection{Neural Network Prediction Model Controller}

Compared with the traditional PID controller, the model prediction control not only use deviation information both the past and present, but also can get the deviation value of the process via the predictive model at future [3]. Therefore, the control effect is better [4].

Predictive Model: Due to the characteristics of the nonlinear system, we adopt neural network to make the predictive model. Model output is:

$y_{m}=O$

$\mathrm{O}$ is the output of neural network, BP neural network weights between each neurons parameters was obtained by the training sample.

Reference Trajectory: Set $y_{d}=\left(\varphi_{d}, \theta_{d}, \psi_{d}\right)^{T}$, reference trajectory is:

$y_{r}(t+k)=(1-\alpha) y_{d}+\alpha y(t)$

Inside, $\alpha=\left(e^{-\frac{T_{s}}{T}}\right)^{k}, T_{s}$ is the sampling period and $\mathrm{T}$ is a time constant of reference trajectory.

Feedback Correction: Use error between measured and predicted quantity to correct model output. With correcting by feedback of historical error, predictive model output is:

$y_{p}(t+k)=y_{m}(t+k)+\beta\left[y(t)-y_{m}(t)\right]$

Rolling Optimization: Optimization is based on real-time manipulation at each sampling time, and get optimal control rule since starting sampling. Actual optimal control law applied to 
controlled obtain is only the current item, the item of next sampling moment is calculated again. According to the optimization algorithm and the optimal control:

$$
J=\sum_{N_{1}}^{N_{z}} e^{2}(t+k)+\mu \sum_{0}^{N_{u}} \Delta u^{2}(t+k)
$$

Inside, $e(t+k)=y_{r}(t+k)-y_{p}(t+k), \Delta u(t+k)=u(t+k)-u(t+k-1)$.The resulting control sequence is $u(t+k)$, this is our controller output.

\subsection{A Cascade Control Structure Block}

There form a dual predictive control method we use neural network predictive model to control respectively the dynamics and kinematics module [5]. Set an output reference angle value given for the second part of kinematics module, the input of the dynamics module in the first part refer from the actual control sequence that is occurred by neural network prediction of the kinematics module in the second part, because this sequence is constantly changing, so the dynamics module reference is dynamic, then apply Dynamics module control input to the real model of the controlled object, establish a cascade controller As shown in the Fig. 1.

Inside, NN Predictive Controller is shown in Fig. 2 and Fig. 3.

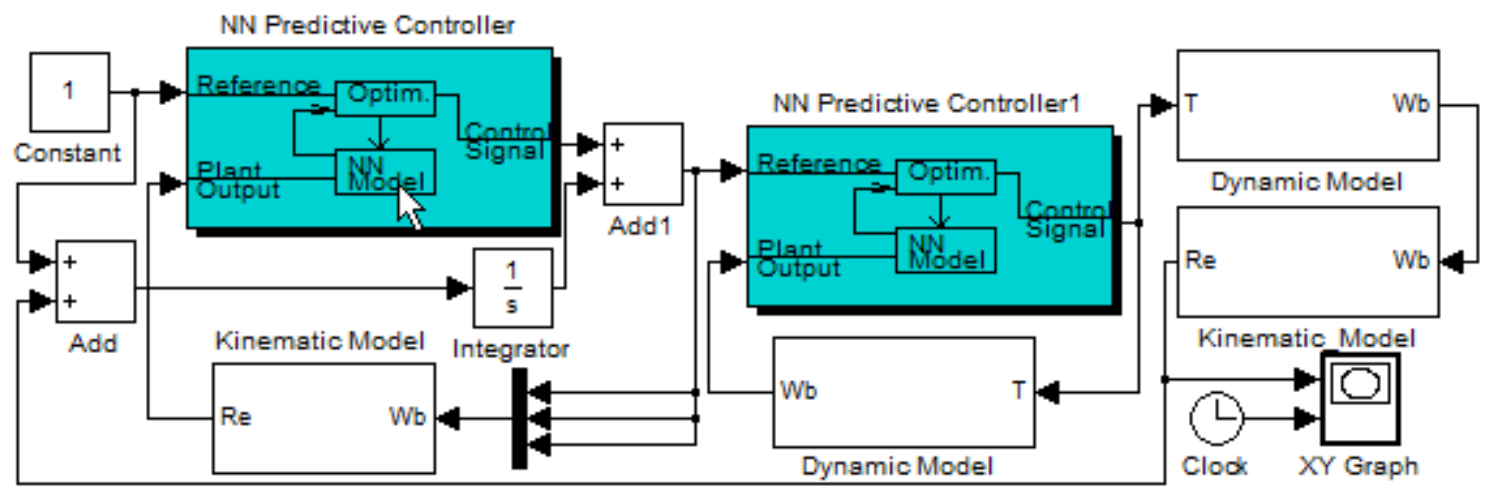

Fig.1 A cascade control structure block diagram of neural network predictive model

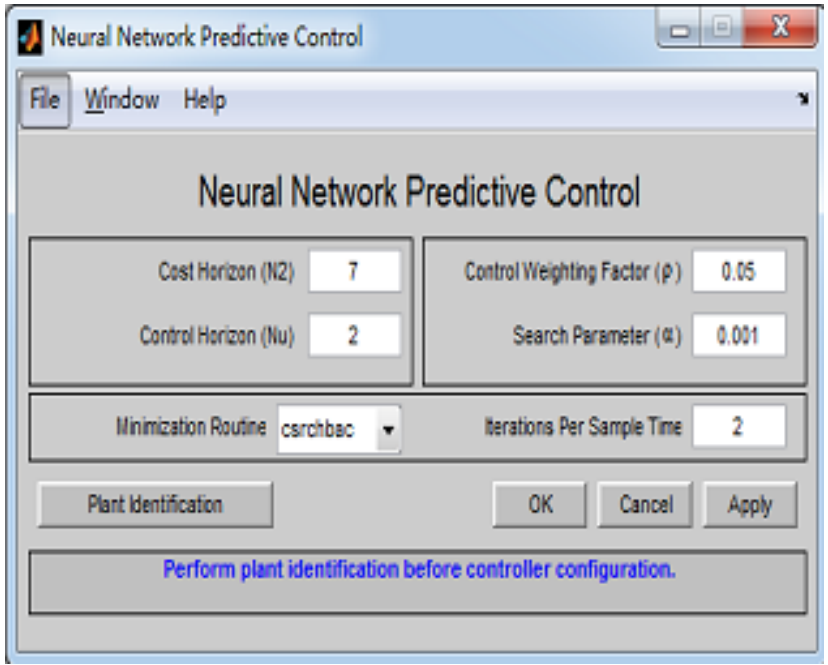

Fig. 2 NN predictive control parameter

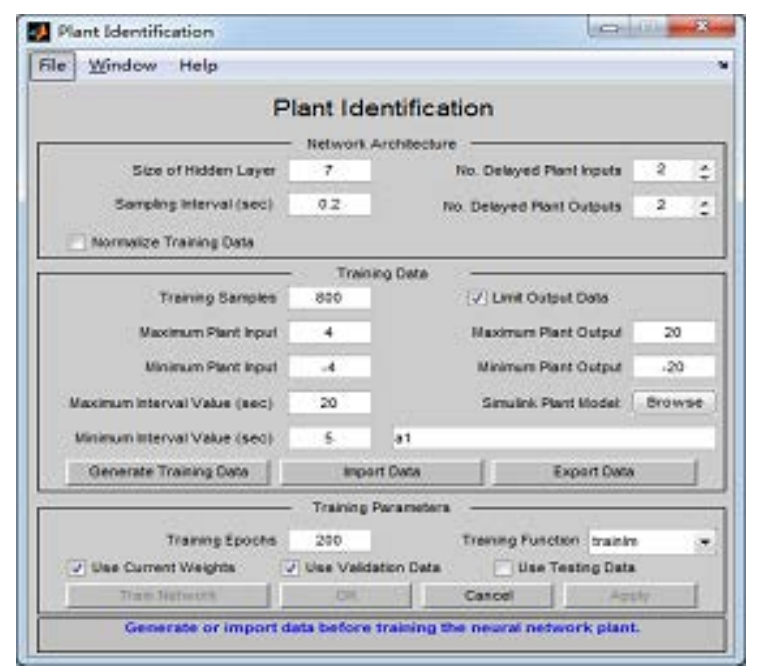

Fig. 3 Plant identification parameter

\subsection{A Three Dimensional PID Controller}

A single dimensional PID controller is used to generate a control effect on the output of the roll angle, pitch angle and yaw angle. The control torque of the three directions are the same, due to the axis of rotation inertia, J1, J2, J3, are not equal to each other, and changes didn't get negative feedback control in the direction of the other two output. Therefore, the integrator input to an illegal value, not only system cannot achieve feedback attitude angle control, but also the trend of feedback attitude angle cannot stabilize the set value, the reason is the coupling effect between the axis. Under the random disturbance signal, the steady state fluctuation in the roll Angle is opposite bigger, shaft coupling also affects the stability of the other axis and persistent oscillation process. 
In order to overcome these drawbacks, we adopt a three-dimensional PID controller, which is the three attitude angles were fed back to the three PID controller, and then the control output value of the each controller is mixed as the total control quantity. When the three attitude angles are equal to the initial value, we can achieve the attitude control perfectly. Control model and control results are shown in figure and diagram. In the process of solving the PID parameters of the ideal 4:1 control output curve, we use the critical ratio method, and we get the parameters: $K p=2, K d=0.375$, $\mathrm{Ki}=0.67$.

\section{Simulation and Analysis}

A signal in Fig. 4 was input to control system with PID, so we acquired output of Roll as shown in Fig. 5, and error curve in Fig. 6. Then interference signal in Fig. 7 was added to control system too, system output is shown in Fig. 8 under disturbance. When controlled quantity shown in Fig. 9 is input to control system with neural network predictive model, we obtain control curve as shown in Fig. 10, Fig. 11, Fig. 12.

While research the output curve of the neural network prediction model, we have the following features: (1). Rise time, adjust time shorter, system response is well; (2). Overshoot less, and even can achieve no overshoot state; (3). Output curve is smooth, there is no steady-state error; (4). There is more stable state under unpredictable disturbances.

But the multistage neural network prediction control increase greatly account of caculation, and there will have big error between the output of the neural network prediction model and the actual in some situation and appear peaks and troughs similar to the shock signal in some cases. Its range of attitude angle controlled stably is less than PID control.

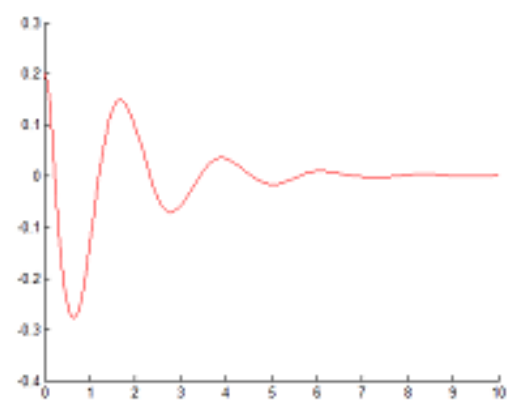

Fig. 4 Control quantity with PID

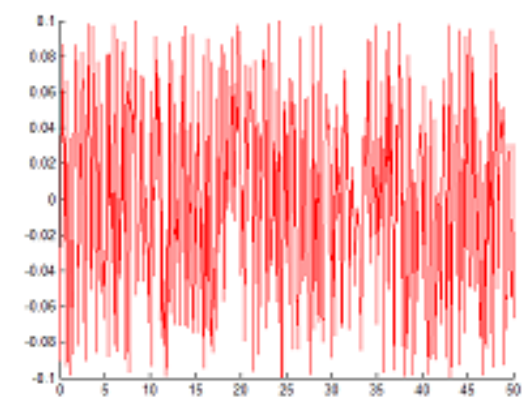

Fig. 7 Interference signal

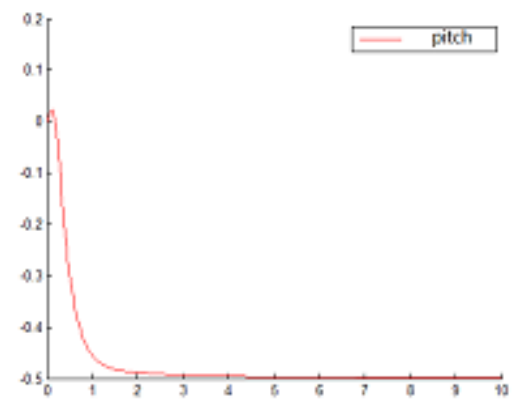

Fig. 10 Pitch curve

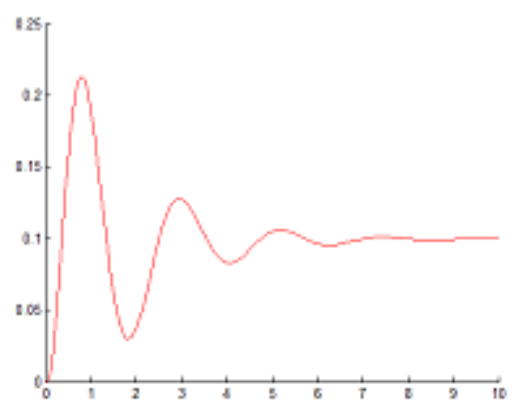

Fig. 5 Roll curve with PID

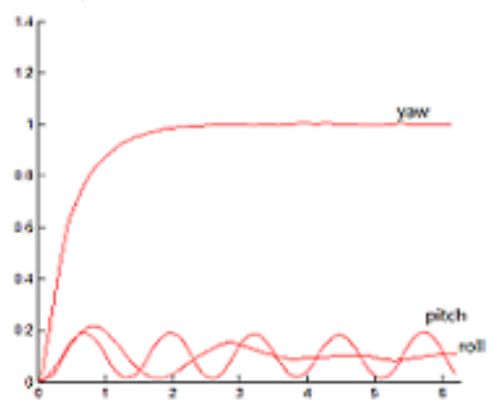

Fig. 8 PID output

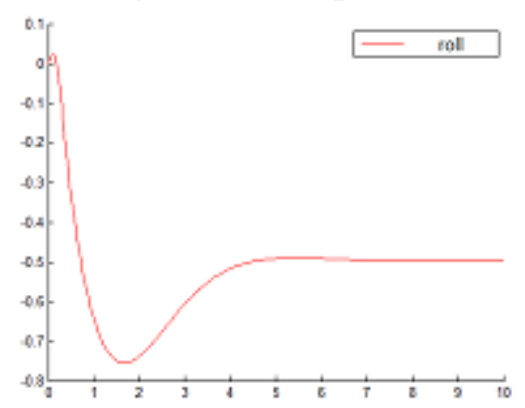

Fig. 11 Roll curve

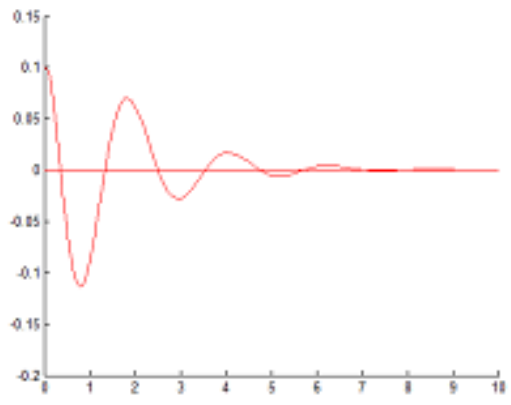

Fig. 6 Error curve with PID

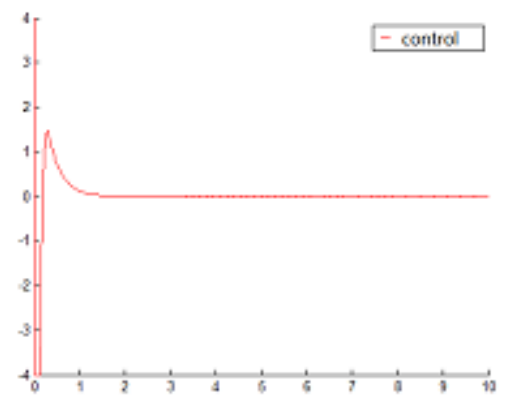

Fig. 9 Controlled quantity

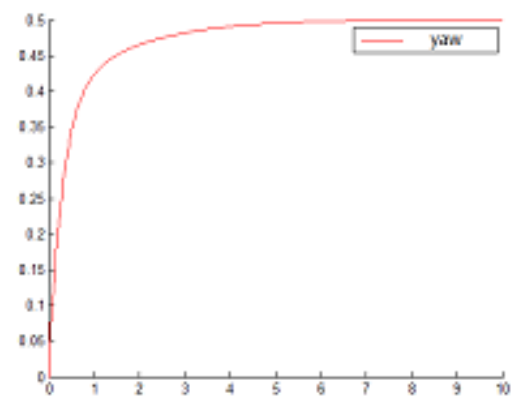

Fig. 12 Yaw curve 


\section{Conclusion}

The attitude control is an important part of UAV control system, it is interdisciplinary science field including air dynamics, the body dynamics, kinematics, transmission signal detection, and many other key fields of science [6]. How to achieve the process of flight attitude control stability and high precision need to consider the coupling nonlinear and unpredictable disturbance factors. This research has been carried out on the basis of the existing research theories and design cases, aiming at the technical difficulties of UAV attitude control. According to theoretical analysis and simulation show that, compared with the classical PID controller, using the neural network prediction model of cascade control system has obvious advantages at the rapidity and optimization of controlled quantity, controlled object still can ensure the stability to some extent under the unknown time-varying torque disturbance.

\section{Acknowledgements}

In this paper, the research was sponsored by the Fundamental Research Funds for the Central Universities (Project No. 06105054).

\section{References}

[1] Mun-Taek Choi and Henryk Flashner. Neural-network-based Spacecarft Attitude Control and Momentum Management. AIAA, 2000, 44-45.

[2] NWE T T, HTIKE T, MON K M. Application of an inertial navigation system to the quad-rotor UAV using MEMS sensors. Proceedings of world academy of science, 2008, 32(8).

[3] Calise A J, Rysdyk R T. Nonlinear Adaptive Flight Control Using Neural Networks [J]. IEEE Control Systems Magazine, 1998, 18(6): 14-25.

[4] Jiang Ping, Rolf Unbehauen. Iterative Learning Neural Network Control for Nonlinear System Trajectory Tracking[J]. Neurocomputing, 2002, 25(48): 141-153.

[5] CASTILLO P, DZUL A, LOZANO R. Real-time stabilization and tracking of a four rotor mini rotorcraft. IEEE transactions on control systems technology, 2004, 12(7).

[6] Jiao Xiaohong, Guan Xinping. Nonlinear System Analysis and Design [M]. Beijing: Publishing House of Electronics Industry, 2008. 